\title{
Primary health clinic bathroom/toilet surface swab sampling can indicate community profile of sexually transmitted infections
}

\author{
Philip M Giffard ${ }^{\text {Corresp., }}{ }^{1,2}$ ， Jiunn-Yih Su ${ }^{3,4}$ ， Patiyan Andersson ${ }^{1}$, Deborah C Holt ${ }^{1}$ \\ 1 Division of Global and Tropical Health, Menzies School of Health Research, Darwin, Northern Territory, Australia \\ 2 School of Psychological and Clinical Sciences, Charles Darwin University, Darwin, Northern Territory, Australia \\ 3 Division of Child Health, Menzies School of Health Research, Darwin, Northern Territory, Australia \\ ${ }^{4}$ Centre for Disease Control, Northern Territory Department of Health, Darwin, Northern Territory, Australia \\ Corresponding Author: Philip M Giffard \\ Email address: phil.giffard@menzies.edu.au
}

Background. The microbiome of built environment surfaces is impacted by the presence of humans. In this study, we tested the hypothesis that analysis of surface swabs from clinic bathroom/toilets yields results correlated with sexually transmitted infection (STI) notifications from corresponding human populations. We extended a previously reported study in which surfaces in toilet/bathroom facilities in primary health clinics in the Australian Northern Territory (NT) were swabbed then tested for nucleic acid from the sexually transmitted infection (STI) agents Chlamydia trachomatis, Neisseria gonorrhoea and Trichomonas vaginalis. This was in the context of assessing the potential for such nucleic acid to contaminate specimens collected in such facilities. STIs are notifiable in the NT, thus allowing comparison of swab and notification data. Methods. An assumption in the design was that while absolute built environment loads of STI nucleic acids will be a function of patient traffic density and facility cleaning protocols, the relative loads of STI nucleic acids from different species will be largely unaffected by these processes. Another assumption was that the proportion of swabs testing positive for STIs provides a measure of surface contamination. Accordingly, "STI profiles" were calculated. These were the proportions that each of the three STIS of interest contributed to the summed STI positive swabs or notifications. Three comparisons were performed, using swab data from clinics in remote Indigenous communities, clinics in small-medium towns, and a single urban sexual health clinic. These data were compared with time and place-matched STI notifications. Results. There was significant correlations between swab and notifications data for the both the remote Indigenous and regional data. For the remote Indigenous clinics the $p$ values ranged from 0.041 to 0.0089 , depending on data transformation and $p$ value inference method. Further, the swab data appeared to strongly indicate known higher relative prevalence of gonorrhoea in central Australia than in northern Australia. Similarly, 
the regional clinics yielded $p$ values from $0.0088-0.0022$. In contrast, swab and notifications data from the sexual health clinic were not correlated. Discussion. Strong correlations between swab and notifications were observed. However, there was evidence for limitations of this approach. Despite the correlation observed with the regional clinics data, one clinic yielded zero positive swabs for C. trachomatis, although this STI constituted $25.1 \%$ of the corresponding notifications. This could be ascribed to stochastic effects. The lack of correlation observed for sexual health clinic data was also likely due to stochastic effects. It was concluded that toilet/bathroom surface swab sampling has considerable potential for public health surveillance. The approach may be applicable in situations other than primary health clinics, and for targets other than STIs. 
1 Primary health clinic bathroom/toilet surface swab sampling can indicate community

2 profile of sexually transmitted infections.

3 Philip M. Giffard ${ }^{1,2}$, Jiunn-Yih Su ${ }^{3,4}$, Patiyan Andersson ${ }^{1}$, Deborah C. Holt ${ }^{1}$

$4{ }^{1}$ Division of Global and Tropical Health, Menzies School of Health Research, Darwin, Northern

5 Territory, Australia.

$6 \quad{ }^{2}$ School of Psychological and Clinical Sciences, Charles Darwin University, Darwin, Northern

7 Territory, Australia

$8{ }^{3}$ Division of Child Health,Darwin, Menzies School of Health Research, Northern Territory,

9 Australia.

$10{ }^{4}$ Centre for Disease Control, Northern Territory Department of Health, Darwin, Northern

11 Territory, Australia.

12 Corresponding author: Philip M. Giffard, phil.giffard@menzies.edu.au 


\section{Abstract}

15 Background. The microbiome of built environment surfaces is impacted by the presence of

16 humans. In this study, we tested the hypothesis that analysis of surface swabs from clinic

17 bathroom/toilets yields results correlated with sexually transmitted infection (STI) notifications

18 from corresponding human populations. We extended a previously reported study in which surfaces in toilet/bathroom facilities in primary health clinics in the Australian Northern Territory (NT) were swabbed then tested for nucleic acid from the sexually transmitted infection (STI) agents Chlamydia trachomatis, Neisseria gonorrhoea and Trichomonas vaginalis. This was in the context of assessing the potential for such nucleic acid to contaminate specimens collected in such facilities. STIs are notifiable in the NT, thus allowing comparison of swab and notification data.

Methods. An assumption in the design was that while absolute built environment loads of STI nucleic acids will be a function of patient traffic density and facility cleaning protocols, the relative loads of STI nucleic acids from different species will be largely unaffected by these processes. Another assumption was that the proportion of swabs testing positive for STIs provides a measure of surface contamination. Accordingly, "STI profiles" were calculated. These were the proportions that each of the three STIs of interest contributed to the summed STI positive swabs or notifications. Three comparisons were performed, using swab data from clinics in remote Indigenous communities, clinics in small-medium towns, and a single urban sexual health clinic. These data were compared with time and place-matched STI notifications.

Results. There was significant correlations between swab and notifications data for the both the remote Indigenous and regional data. For the remote Indigenous clinics the p values ranged from 
360.041 to 0.0089 , depending on data transformation and $\mathrm{p}$ value inference method. Further, the

37 swab data appeared to strongly indicate known higher relative prevalence of gonorrhoea in

38 central Australia than in northern Australia. Similarly, the regional clinics yielded $\mathrm{p}$ values from

$39 \quad 0.0088-0.0022$. In contrast, swab and notifications data from the sexual health clinic were not

40 correlated.

41 Discussion. Strong correlations between swab and notifications were observed. However, there

42 was evidence for limitations of this approach. Despite the correlation observed with the regional

43 clinics data, one clinic yielded zero positive swabs for C. trachomatis, although this STI

44 constituted $25.1 \%$ of the corresponding notifications. This could be ascribed to stochastic effects.

45 The lack of correlation observed for sexual health clinic data was also likely due to stochastic

46 effects. It was concluded that toilet/bathroom surface swab sampling has considerable potential

47 for public health surveillance. The approach may be applicable in situations other than primary

48 health clinics, and for targets other than STIs. 


\section{Introduction}

Recent microbiome and metagenome-based analyses support the notion that the surface microbiomes of human inhabited built environments are closely connected to the human microbiome (Afshinnekoo et al. 2015; Flores et al. 2011; Hsu et al. 2016; Lax et al. 2014; Meadow et al. 2014; Schaumburg et al. 2016). This raises the possibility that microbiological analysis of built environment surfaces could provide information of public health utility; for example the surface may serve as a sentinel for microbial species, strains, genes or gene variants of interest. The advantages of such an approach to surveillance are that it is potentially simple and cost effective, and the specimens can be obtained without any burden upon or identification of individual patients or research participants.

Recently, a longitudinal multi-site assessment of sexually transmitted infection (STI) agent nucleic acid contamination of surfaces in clinic toilet/bathroom facilities in the Australian Northern Territory (NT) was reported (Andersson et al. 2014). The aim was estimation of the potential for such contamination to lead to positive STIs tests performed on urine specimens, so the study also included simulation of urine specimen collection using a synthetic urine substitute. The STIs targeted were Chlamydia trachomatis, Neisseria gonorrhoea and Trichomonas vaginalis. Surface contamination with STI nucleic acid was found in all clinics except for the sexual assault clinics, with the heaviest contamination in the remote primary health clinics. The frequency of simulated urine specimens testing positive for STIs was low but non-zero. Other studies in the UK have shown that STI agent nucleic acid contamination of clinic surfaces can readily be detected (Lewis et al. 2012; Meader et al. 2008). 
71 In the NT, STIs are notifiable, and notifications are placed in the public domain in the form of

72 de-identified and aggregated data reported quarterly with some geographic breakdown (Northern

73 Territory Department of Health). We reasoned that availability of time and locality matched

74 toilet/bathroom surface swabbing data STI notifications data provided a unique and compelling

75 opportunity to determine whether the results from toilet-bathroom swabbing are predictive of

76 notifications. Surface cleaning protocols in clinic toilet/bathroom facilities will potentially be

77 highly variable, and this would be expected to impact upon absolute STI nucleic acid load.

78 Therefore, we did not regard it as useful to test the conjecture that absolute STI nucleic acid load

79 on clinic toilet-bathroom surfaces predicts absolute STI prevalence in the community. Rather, the

80 experimental design was based on the premise that while toilet/bathroom cleaning protocols

81 would be likely to greatly impact absolute STI nucleic acid prevalence on toilet/bathroom

82 surfaces, they would have much less impact on the relative loads of nucleic acid from different

83 STI agents. Therefore, the conjecture we tested was that the relative prevalences of different STI

84 agents in clinic toilet/bathroom swabs would be correlated with the relative prevalences of these

85 STIs in the notifications data.

\section{Methods}

87 The basis of this study was the comparison of previously published data concerning STI agents

88 in toilet/bathroom surface swabs (Andersson et al. 2014) with corresponding NT Government

89 STI notifications data (Northern Territory Department of Health). The detailed and definitive

90 procedure for the swab collection and analysis is described by Andersson and co-

91 workers(Andersson et al. 2014). In summary, seven sites within the NT were included: two

92 clinics associated with an NT Government organisation that investigates possible sexual assaults,

93 two "sexual health" clinics in the Darwin and Alice Springs urban areas, two primary health 
94 clinics in small "regional" towns, and four primary health clinics in "remote Indigenous"

95

96

97

98

communities. Each site was visited seven times over a period of approximately 12 months, and at each visit a standardised set of 10 toilet/bathroom surfaces were swabbed. The surfaces encompassed the floor, walls, toilet components, wash basin components and the door handle. At each visit, the experiment was performed in the male and female toilet/bathrooms. The swabs were subjected to the same STI diagnostic procedure as actual clinical specimens for STI analysis from the clinic concerned i.e. the swabs were stored, transported to a diagnostic service provider, and subjected to C. trachomatis, $N$. gonorrhoea, and T. vaginalis diagnostic procedures, along with actual STI diagnostic specimens generated on that day by the clinic concerned. Swabs from the sexual assault and sexual health clinic were analysed using the DNAbased Versant CT/GC DNA 10 Assay (kPCR) (Siemens), and swabs from the regional and remote clinics were analysed by a different service provider who used the RNA-based APTIMA Combo 2 Assay system (Gen-Probe). The results of the swab analysis are presented in detail in Tables 1-3 of the paper by Andersson and co-workers (Andersson et al. 2014).

The current study encompassed swab data from the remote Indigenous and regional clinics, and the Darwin sexual health clinic. Two of the remote Indigenous clinics were located in the tropical 'Top End" of the NT (close to the north coast), and swab data from these were compared with STI notifications data from the NT Government-defined "East Arnhem" region. The other two remote Indigenous clinics were located in Central Australia and the swab data were compared with "Alice Springs rural” STI notifications data. The numbers of positive swabs were normalised because of variation from the default number of 140 tests per STI agent per clinic. This variation was due to rare failure of service providers to complete tests. For example, for one remote clinic, 31 of 119 tested swabs were positive for T. vaginalis, and this was normalised to 
11735.3 positive swabs from 140 (Andersson et al. 2014). The swab and notifications data were

118 compared on the basis of "STI profiles" comprised of the proportions contributed by each of

119 C. trachomatis, $N$. gonorrhoea and T. vaginalis positive tests/notifications to total positive

120 swabs/notifications for these species.

121 The same approach was used to compare swab and notifications data from regional clinics.

122 These are in towns that are not Indigenous communities, but have substantial Indigenous

123 populations. In accordance with location, the swab data from one regional clinic was compared

124 with notifications data from the Barkly region of the NT and the other compared with

125 notifications data from the NT East Arnhem region. Finally, the same approach was used to

126 compare swabbing and notifications data from a sexual health clinic located in Darwin.

127 The notifications data used were from 2012 (Northern Territory Department of Health 2012a;

128 Northern Territory Department of Health 2012b). The great majority of the swabbing was

129 performed in 2012, with a minority of the swabbing for a subset of clinics performed in late 2011

130 or early 2013 (Andersson et al. 2014). There were no major changes in STI relative prevalences

131 in notifications data from late 2011 to early 2012, or from late 2012 to early 2013 (Northern

132 Territory Department of Health), so we regarded comparing all swab data with 2012 notifications

133 as justified. All the time and place specific notifications data were included in down-loadable

134 documents in the public domain, (Northern Territory Department of Health 2012a; Northern

135 Territory Department of Health 2012b) with the exception of the notifications data for the

136 Darwin sexual health clinic for which a custom data extraction was performed.

137 Correlations were assessed using the Pearson Correlation Coefficient $(r)$ for linear regressions of 138 the swab and notifications proportions data. The logit transformation $(\log (\mathrm{x} /(1-\mathrm{x})))$ and arcsine 
139 transformation $(\arcsin (\sqrt{ } \mathrm{x}))$ are reported to be useful in statistical analyses of proportions, with

140 the logit regarded as superior, but not able to be used with proportions of 0 or 1 (Warton \& Hui

141 2011). In order to comprehensively explore the association between swab and notifications data,

142 untransformed, logit transformed and arcsine transformed data were used. In addition, we

143 minimised the requirement for assumptions regarding data distribution by inferring $p$ values

144 using a permutation approach. We developed macros in Microsoft Excel that for each data set

145 performed 5000 iterative randomisations of the order of one data column and recalculation of $r$.

146 The inferred $p$ value was the proportion of iterations for which $\mathrm{r}$ (randomised data) $>\mathrm{r}$ (non-

147 randomised data, expressed with fewer decimal places than calculated values of $r$, and rounded

148 down). $p$ values were also calculated using the $t$ statistic (Soper 2017).

149 Ethical clearance for study was provided by the Human Research Ethics Committee of the

150 Northern Territory Department of Health and the Menzies School of Health Research. The

151 permit is HREC 2016-2696, "Environmental contamination as a predictor of community sexually

152 transmitted infections profile"

\section{Results}

154 First, we analysed data from 420 toilet/bathroom surface swabs from remote Indigenous communities. This experiment was of particular interest because these clinics generated the

156 highest proportion and number of STI positive swabs, maximising statistical power. Also, remote

157 primary health clinics are broadly similar in their characteristics and client base throughout the

158 NT, but the notifications data indicated that the relative prevalence of $N$. gonorrhoea was much

159 higher in Alice Springs rural than in East Arnhem at the time the swabbing was performed,

160 which we predicted would be reflected in swab data. From the tests for three STI agents on the 
161420 swabs, 208 positive results were obtained (Andersson et al. 2014). The STI profile defined

162 by these results was compared with the breakdown of time and place-matched STI notifications

163 as described in the Methods (Fig 1, Table 1). There was statistical support for correlation of STI

164 profiles. For all three transformations of the data, the permutation method yielded a higher (i.e.

165 inferior) $p$ value than the t-statistic method, but still indicated $\mathrm{p}$ values $<0.05$ (Table 1 ). The $p$

166 values from the logit and arcsin transformed data were both lower (i.e. superior) than from the

167 untransformed data (Table 1). The $N$. gonorrhoea data were of particular interest because

$168 N$. gonorrhoea notifications are a much smaller proportion of the total in East Arnhem than in

169 Alice Springs Rural (Northern Territory Department of Health 2012a; Northern Territory

170 Department of Health 2012b). The correlation of this with the swabbing data is striking.

$171 N$. gonorrhoea contributes $4.6 \%$ of the positive swabs and $9.5 \%$ of the notifications from the Top

172 End/East Arnhem, and $41.5 \%$ of the positive swabs and $46.1 \%$ of the notifications from Central

173 Australia/Alice Springs Rural. Complete numeric data are provided as supplementary

174 information.

175 For the regional clinics, 34 positive STI tests were derived from 280 swabs. The swab results

176 from the two clinics were very similar to each other in that the great majority $(30 / 34,88 \%)$ of

177 positive tests were for T. vaginalis. Despite the smaller number of positive tests, the correlation

178 between swabs and notifications was stronger than for the remote clinics (Fig 1, Table 1).

179 Interestingly, the arcsin transformation improved the $p$ value derived from the permutation

180 method but had the opposite effect on the $p$ value derived using the $\mathrm{t}$ statistic. However, as with

181 the Remote Indigenous data, the $p$ values from the permutation method indicated weaker

182 significance than the $p$ values derived using the $t$ statistic. 
183 For the Darwin sexual health clinic, the number of positive STI tests was lower still, with 10

184 positives from 140 swabs. Nine of the ten positive swabs were positive for $N$. gonorrhoea, and

185 with the remaining swab positive for T. vaginalis. This does not correlate with the notifications,

186 for which the majority are C. trachomatis (Fig 1). To explore possible reasons for the lack of

187 correlation, we examined the raw data from the swabbing study (Andersson et al. 2014) and

188 determined that five of the nine positive $N$. gonorrhoea positive swabs were from a single

189 toilet/bathroom (male), on a single day, and two of the remaining positive swabs also coincided

190 in time and place of origin. Therefore we can account for these $9 \mathrm{~N}$ gonorrhoea positive swabs

191 with just four surface contamination events.

192 We also analysed the data from the remote and regional clinics together. All $p$ values obtained

193 were $\leq 0.0024$, and were less than $2 \times 10^{-4}$ when all 12 points could be included (untransformed

194 and arcsin transformed data) (Table 1), thus providing additional support that the correlation

195 between the swab and notifications is significant (Fig 1).

\section{Discussion}

197 This study illustrates both the potential value and some limitations of surface swabbing of clinic toilet/bathroom facilities for inferring STI profile in the community. We predicted that correlation would be highest with data from the remote clinics, because the large number of

200 positive swabs (205) would minimise sampling error. Consistent with this, clear correlation was observed, and it appeared that the difference in relative prevalence of $N$. gonorrhoea in remote central Australia and East Arnhem was reflected in the swab data.

Somewhat unexpectedly, the correlation from the "Regional" experiment was better than for the 
205 close examination of the data from the regional clinics does suggest that stochastic effects are

206 evident. While the swab data are consistent with $T$. vaginalis having the highest relative

207 prevalence in the relevant populations for both regional clinics, zero swabs from one of the

208 clinics were positive for C. trachomatis or N. gonorrhoea, despite these STIs representing $25.1 \%$

209 and $10.4 \%$ of the notifications in the region used for the comparison. Similarly for the other

210 regional clinic, zero swabs were positive for $N$. gonorrhoea, despite this representing $24.9 \%$ of

211 the notifications used for the comparison. Thus the swab data from the regional clinics has failed

212 to indicate the presence of STIs that comprise sizeable proportions of the notifications data.

213 The swab data from the sexual health clinic failed to reveal any useful information regarding the

214 relative prevalances of STIs diagnosed in the same clinic. A clear anomaly is the lack of swabs

215 positive for $C$. trachomatis, despite these being constituting $79.2 \%$ of the notifications. The basis

216 for this is unknown, but we speculate it is related to frequent cleaning of toilet bathrooms in this

217 facility. This begs the question as to why N. gonorrhoea positive swabs were obtained. As stated

218 in the "Results", five of the nine $N$. gonorrhoea positive swabs arose from a single swabbing

219 visit to a single male toilet/bathroom, highly suggestive of a single contamination event

220 accounting for the majority of the $N$. gonorrhoea positive swabs. It is also noteworthy that the

221 simulated urine collection procedure at that time and place generated an $N$. gonorrhoea positive

222 specimen (Andersson et al. 2014). Positive results from the simulated urine collection

223 experiments were very rare in that study as whole, so this is suggestive of a single very

224 significant contamination event. It is of interest that the swab data from the Alice Springs sexual

225 health clinic were very similar, with seven positive swabs for N. gonorrhoea, and one positive

226 swab for each of $T$. vaginalis and C. trachomatis. Because a DNA diagnostic technology was

227 used for the sexual health clinic swabs and RNA based method for the other swabs encompassed 
228 by this study, we cannot rule out that STI diagnostic method affects the correlation between swab 229 data and notifications data. While we regard this as unlikely, primarily because of the robustness 230 and intensive quality control of commercial STI diagnostic systems, this may be an area worthy 231 of future study.

232 We examined the data for other associations of interest. It was previously noted that positive swabs from female toilets are much more likely to be positive for T. vaginalis than swabs from male toilets, while the reverse is true for $N$. gonorrhoea (Andersson et al. 2014). This is consistent with known disease processes, with T. vaginalis infection generally being regarded as more likely to cause symptoms and discharge in women than in men (Muzny \& Schwebke

237 2013), and N. gonorrhoea being usually asymptomatic in women (Mayor et al. 2012).

Examination of the primary data from the surface swabbing study revealed that this bias is most extreme in the regional and sexual health clinics, with all $34 T$. vaginalis positive swabs from regional clinics being from female toilets, and all 16 of the $N$. gonorrhoea positive swabs from the sexual health clinics being from male toilets. It should be noted however that the single T. vaginalis swab from a sexual health clinic was from a male toilet/bathroom, and that association of $N$. gonorrhoea with male toilets in the sexual health clinics may be related to the strong association of $N$. gonorrhoea with men who have sex with men in the non-Indigenous population of Australia (The Kirby Institute 2015). There were no N. gonorrhoea positive swabs 246 from regional clinics.

247 Our intention in conducting this study was not primarily to foreshadow implementation of toilet/bathroom surface swabbing for routine STI surveillance in the NT. There is no evidence that the STI notification system in the NT is ineffective. Indeed it is the availability of 
251 the swabbing approach. We suggest that toilet/bathroom surface swabbing may have most

252 potential utility in resource poor environments where there is no effective system of STI

253 notification. However, we would not rule out toilet/bathroom swabbing could provide valuable

254 information that is complementary to notifications data. A surface swabbing approach to STI

255 surveillance encompasses people who have not been subjected to STI diagnostic procedures, and

256 it may have potential to provide early indications of outbreaks. Possible applications of this

257 approach may be in environments other than clinics, such as entertainment precincts or travel

258 hubs. A built environment surface swabbing approach could also be very valuable in surveillance

259 for targets other than STI agents, e.g. antibiotic resistance genes or exotic viruses.

260 In conclusion, the surface swabbing in the clinics in the remote communities in the NT was

261 effective at indicating community STI profile, in large part because of the large number of ST

262 positive swabs. It does appear however that different results will potentially be obtained from

263 male and female toilets. While the expected association was also found for the "Regional"

264 clinics, STI agents corresponding with STIs that formed an appreciable proportion of the

265 notifications were not found in swabs. In the sexual health clinic, there was no correlation

266 observed. We concluded that, as would be expected, this approach to surveillance can be

267 confounded by low numbers of STI agent-positive swabs, leading to stochastic effects.

\section{Acknowledgements}

269 The authors thank Peter Markey (Centre for Disease Control, Northern Territory Department of

270 Health, Australia) for providing sexual health clinic specific notifications data, and David

271 Whiley (Centre for Clinical Research, University of Queensland) for helpful discussions. 
273

274

275

276

277

278

279

280

281

282

283

284

285

286

287

288

289

290

291

292

293

294

\section{References}

Afshinnekoo E, Meydan C, Chowdhury S, Jaroudi D, Boyer C, Bernstein N, Maritz JM, Reeves D, Gandara J, Chhangawala S, Ahsanuddin S, Simmons A, Nessel T, Sundaresh B, Pereira E, Jorgensen E, Kolokotronis SO, Kirchberger N, Garcia I, Gandara D, Dhanraj S, Nawrin T, Saletore Y, Alexander N, Vijay P, Henaff EM, Zumbo P, Walsh M, O'Mullan GD, Tighe S, Dudley JT, Dunaif A, Ennis S, O'Halloran E, Magalhaes TR, Boone B, Jones AL, Muth TR, Paolantonio KS, Alter E, Schadt EE, Garbarino J, Prill RJ, Carlton JM, Levy S, and Mason CE. 2015. Geospatial Resolution of Human and Bacterial Diversity with City-Scale Metagenomics. Cell Syst 1:97-97 e93.

10.1016/j.cels.2015.07.006

Andersson P, Tong SY, Lilliebridge RA, Brenner NC, Martin LM, Spencer E, Delima J, Singh G, McCann F, Hudson C, Johns T, and Giffard PM. 2014. Multisite Direct Determination of the Potential for Environmental Contamination of Urine Samples Used for Diagnosis of Sexually Transmitted Infections. J Pediatric Infect Dis Soc 3:189-196.

10.1093/jpids/pit085

Flores GE, Bates ST, Knights D, Lauber CL, Stombaugh J, Knight R, and Fierer N. 2011. Microbial biogeography of public restroom surfaces. PLoS One 6:e28132. 10.1371/journal.pone.0028132

Hsu T, Joice R, Vallarino J, Abu-Ali G, Hartmann EM, Shafquat A, DuLong C, Baranowski C, Gevers D, Green JL, Morgan XC, Spengler JD, and Huttenhower C. 2016. Urban Transit System Microbial Communities Differ by Surface Type and Interaction with Humans and the Environment. mSystems 1. 10.1128/mSystems.00018-16 
295

296

297

298

299

300

301

302

303

304

305

306

307

308

309

310

311

312

313

314

315

316

317

Lax S, Smith DP, Hampton-Marcell J, Owens SM, Handley KM, Scott NM, Gibbons SM, Larsen P, Shogan BD, Weiss S, Metcalf JL, Ursell LK, Vazquez-Baeza Y, Van Treuren W, Hasan NA, Gibson MK, Colwell R, Dantas G, Knight R, and Gilbert JA. 2014. Longitudinal analysis of microbial interaction between humans and the indoor environment. Science 345:1048-1052. 10.1126/science.1254529

Lewis N, Dube G, Carter C, Pitt R, Alexander S, Ison CA, Harding J, Brown L, Fryer J, Hodson J, and Ross J. 2012. Chlamydia and gonorrhoea contamination of clinic surfaces. Sex Transm Infect 88:418-421. sextrans-2012-050543 [pii]

$10.1136 /$ sextrans-2012-050543

Mayor MT, Roett MA, and Uduhiri KA. 2012. Diagnosis and management of gonococcal infections. Am Fam Physician 86:931-938.

Meader E, Waters J, and Sillis M. 2008. Chlamydia trachomatis RNA in the environment: is there potential for false-positive nucleic acid amplification test results? Sex Transm Infect 84:107-110.

Meadow JF, Altrichter AE, Kembel SW, Moriyama M, O'Connor TK, Womack AM, Brown GZ, Green JL, and Bohannan BJ. 2014. Bacterial communities on classroom surfaces vary with human contact. Microbiome 2:7. 10.1186/2049-2618-2-7

Muzny CA, and Schwebke JR. 2013. The clinical spectrum of Trichomonas vaginalis infection and challenges to management. Sex Transm Infect 89:423-425. 10.1136/sextrans-2012050893

Northern Territory Department of Health. Northern Territory Sexual Health and Blood Borne Viruses Unit Surveillance Updates. Available at http://hdl.handle.net/10137/237 (accessed May 12017 ). 
318 Northern Territory Department of Health. 2012a. Northern Territory Sexual Health and Blood 319 Borne Viruses Unit Surveillance Update January-March \& April-June 2012. Available at http://digitallibrary.health.nt.gov.au/prodjspui/bitstream/10137/237/101/Surveillance\%2 OUpdate\%20Vol\%2013\%20no\%201\%20January-March\%20\%26\%20April-

Northern Territory Department of Health. 2012b. Northern Territory Sexual Health and Blood Borne Viruses Unit Surveillance Update July-September October-December 2012.

Warton DI, and Hui FK. 2011. The arcsine is asinine: the analysis of proportions in ecology.

Soper DS. 2017. p-Value Calculator for Correlation Coefficients. Available at http://www.danielsoper.com/statcalc/calculator.aspx?id=44 (accessed March 14 2017).

The Kirby Institute. 2015. HIV, viral hepatitis and sexually transmissible infections in Australianual Surveillance Report, 2015 The Kirby Institute, UNSW Australia, Sydney NSW 2052. 
341 Table 1. Correlation coefficients $(r)$ and $\mathrm{p}$ values $(p)$ for STI profiles derived from swab and 342 notifications data. The $p$ values are single tailed. $p$ (perm): $p$ values derived from a permutation 343 test. $\mathrm{p}(\mathrm{t}): \mathrm{p}$ values derived from $\mathrm{t}$ statistic.

\begin{tabular}{|c|c|c|c|c|c|c|c|c|c|}
\hline & \multicolumn{3}{|c|}{ untransformed } & \multicolumn{3}{|c|}{ logit } & \multicolumn{3}{|c|}{$\arcsin$} \\
\hline clinics & $r$ & $\begin{array}{c}p \\
\text { (perm) }\end{array}$ & $p(\mathrm{t})$ & $r$ & $\begin{array}{c}p \\
\text { (perm) }\end{array}$ & $p(\mathrm{t})$ & $r$ & $\begin{array}{c}p \\
\text { (perm) }\end{array}$ & $p(\mathrm{t})$ \\
\hline Remote & 0.857 & 0.041 & 0.015 & 0.891 & 0.015 & 0.0086 & 0.869 & 0.019 & 0.012 \\
\hline Regional & 0.945 & 0.0088 & 0.0022 & ND* & ND* & ND* & 0.924 & 0.0048 & 0.0042 \\
\hline Both & 0.898 & $\begin{array}{l}<2.0 \mathrm{x} \\
10^{-4}\end{array}$ & $\begin{array}{l}3.66 \mathrm{x} \\
10^{-5}\end{array}$ & $0.887 * *$ & $0.0024 * *$ & $0.0016^{* *}$ & 0.870 & $\begin{array}{l}<2.0 \mathrm{x} \\
10^{-4}\end{array}$ & $\begin{array}{l}1.17 \mathrm{x} \\
10^{-4}\end{array}$ \\
\hline
\end{tabular}

344

*'Not Done". These were not done because four of the six points from regional clinics

be performed on $0 \%$ or $100 \%$ values.

**Calculated from all remote clinic data and the two regional clinic points that do not encompass $0 \%$ or $100 \%$ proportions in the swab data. 
Figure legend

$359 \quad$ Fig 1.

360 Correlations of STI profiles indicated by STI notifications and toilet/bathroom swab data. The axes represent percent of total C. trachomatis $+N$. gonorrhoea $+T$. vaginalis notifications/swabs. Where data points are labelled, the first or only letter indicates the organism, "C": C. trachomatis; "G": N. gonorrhoea;" "T": T. vaginalis. The second pair of letters (where present) indicates location, "TE": Top End"; "CE” Central Australia; "BA”: Barkly; "EA": East

367 Regional data.

368

369 Fig $1 \mathrm{~A}$

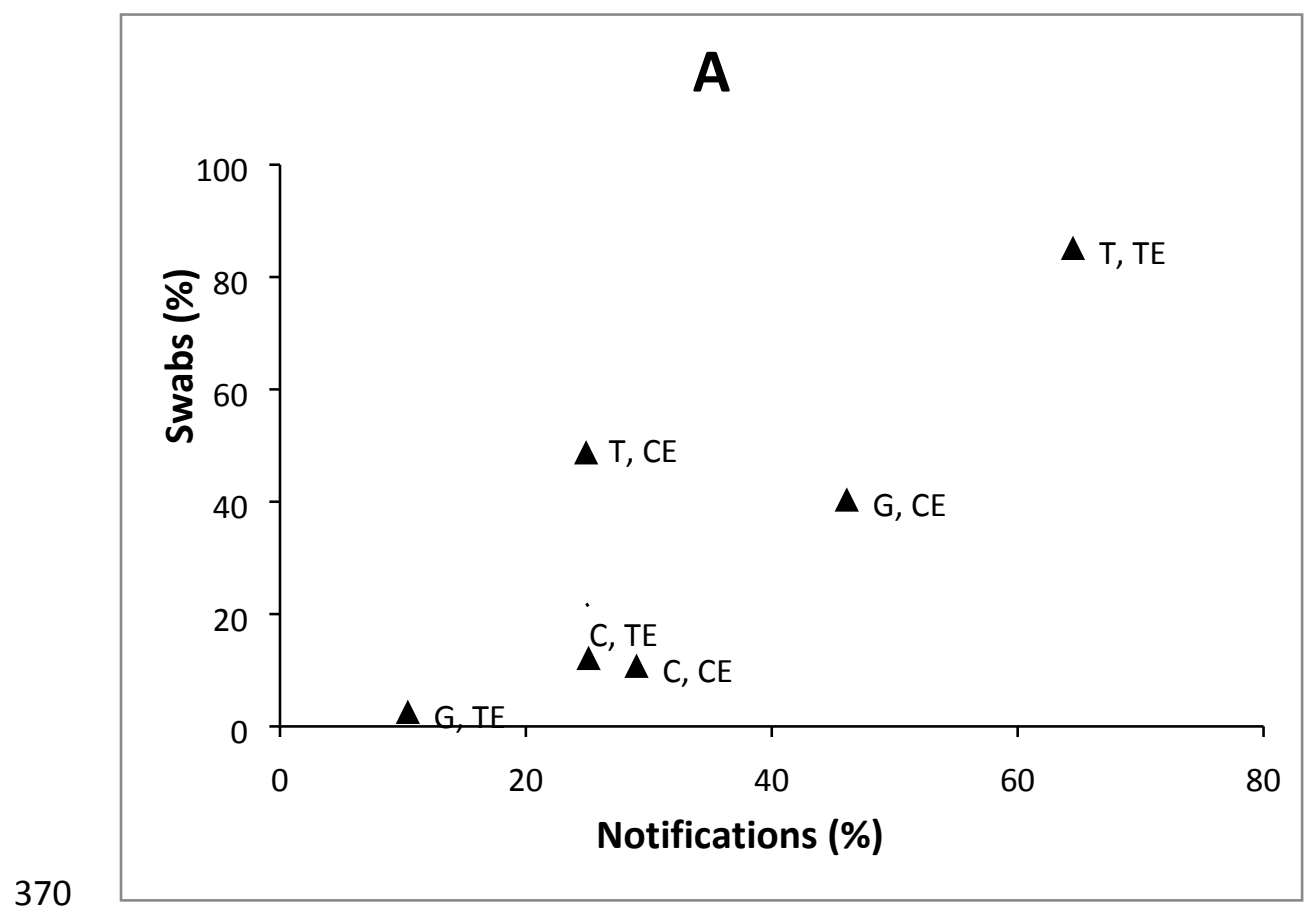


373 Fig1B

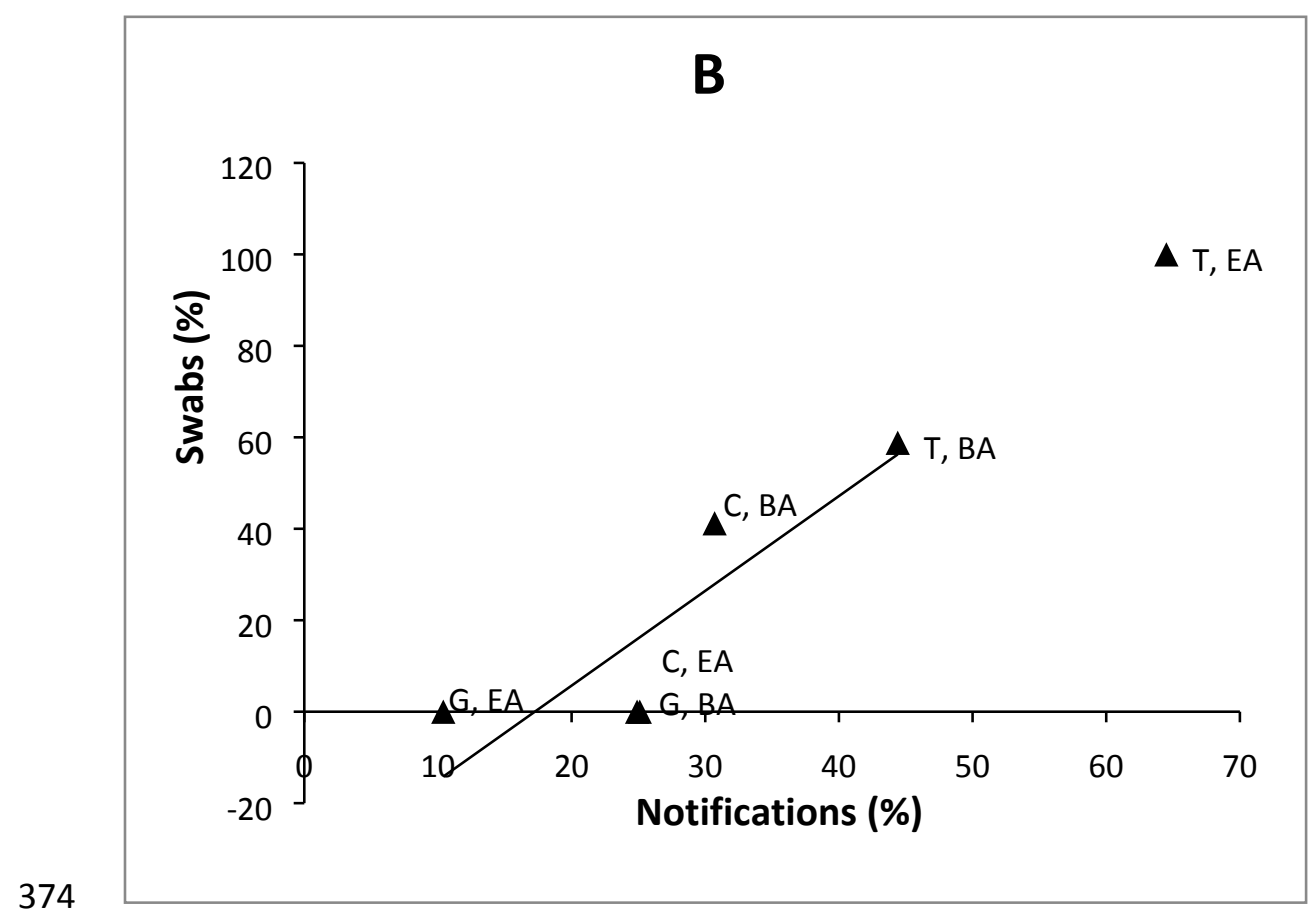

375

376 
377 Fig 1C

378

379

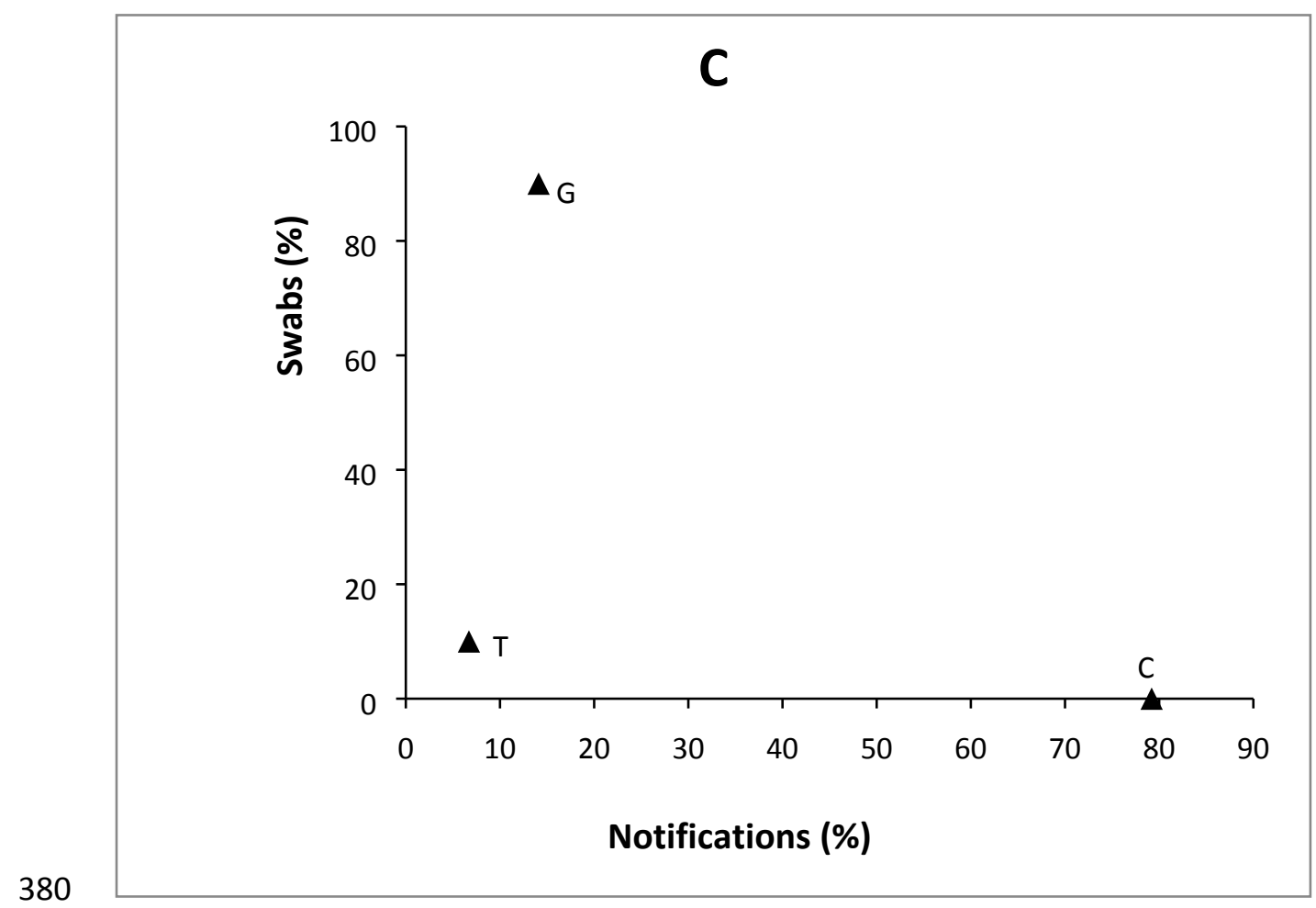

381

382 
383 Fig 1D.

384

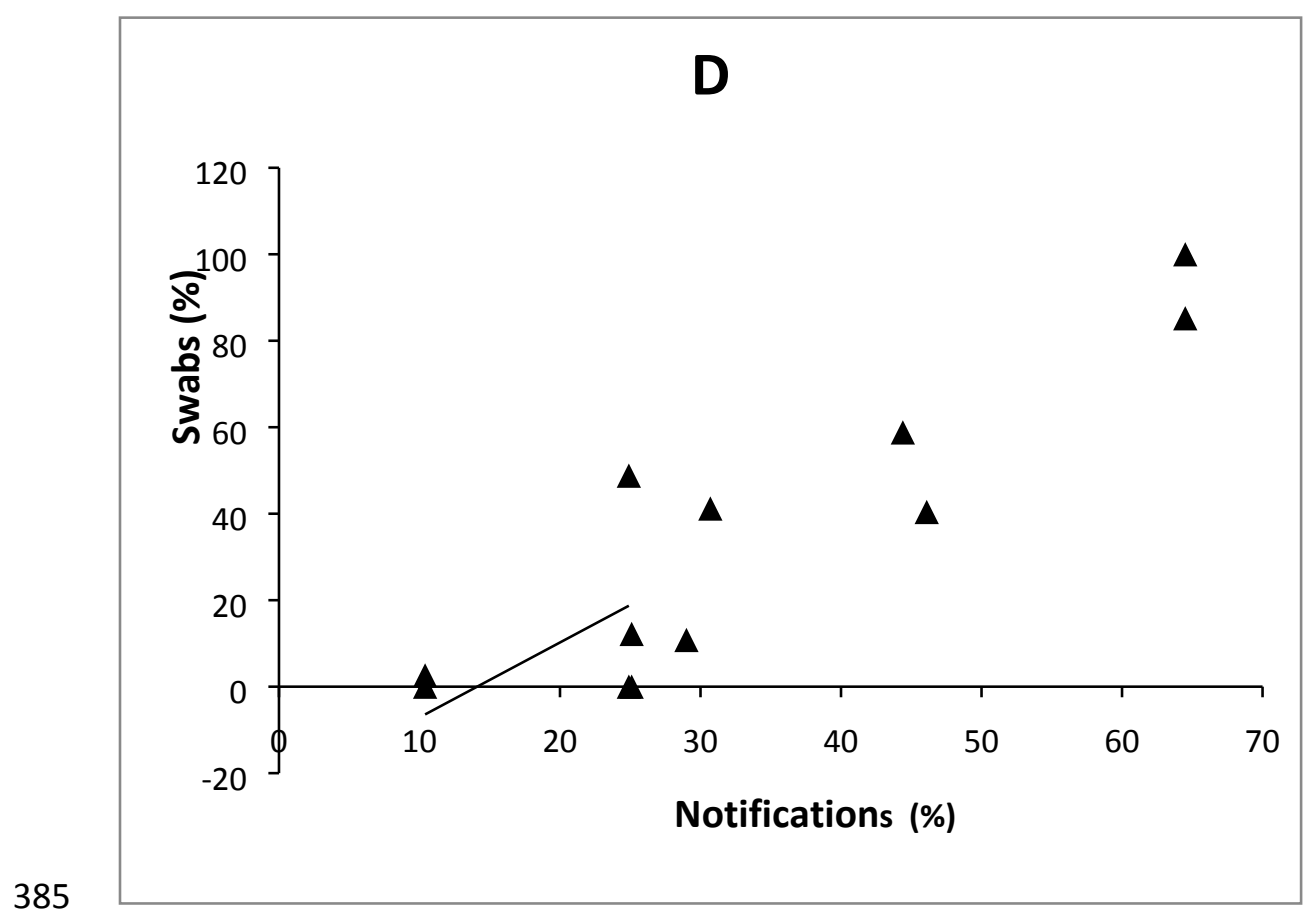

386

387

388

389

390

391

392

393

394

395

396

397

398 
